\title{
Anti-inflammatory and anti-oxidative effects of corilagin in a rat model of acute cholestasis
}

\author{
Feng $\mathrm{Jin}^{1+}{ }^{\dagger}$, Du Cheng ${ }^{2 \dagger}$, Jun-Yan Tao ${ }^{3}$, Shu-Ling Zhang ${ }^{4}$, Ran Pang ${ }^{4}$, Yuan-Jin Guo ${ }^{5}$, Pian Ye ${ }^{4}$, Ji-Hua Dong ${ }^{6}$ \\ and Lei Zhao ${ }^{4^{*}}$
}

\begin{abstract}
Background: Nowadays, treatments for cholestasis remain largely nonspecific and often ineffective. Recent studies showed that inflammatory injuries and oxidative stress occur in the liver with cholestasis. In this study, we would use corilagin to treat the animal model of acute cholestasis in order to define the activity to interfere with inflammation-related and oxidative stress pathway in cholestatic pathogenesis.
\end{abstract}

Methods: Rats were administrated with alpha-naphthylisothiocyanate to establish model of cholestasis and divided into corilagin, ursodeoxycholic acid, dexamethasone, model and normal groups with treatment of related agent. At $24 \mathrm{~h}, 48 \mathrm{~h}$ and $72 \mathrm{~h}$ time points after administration, living condition, serum markers of liver damage, pathological changes of hepatic tissue, nuclear factor (NF)-kappaB, myeloperoxidase (MPO), malondialdehyde (MDA), superoxide dismutase (SOD) and nitric oxide (NO) were examined and observed.

Results: Compared to model group, corilagin had remarkable effect on living condition, pathological manifestation of liver tissue, total bilirubin, direct bilirubin, $(P<0.01)$, but no effect on alanine aminotransferase (ALT) and aspartate aminotransferase (AST). With corilagin intervention, levels of MPO, MDA and translocation of NF-KB were notably decreased, and levels of SOD and NO were markedly increased $(P<0.05$ or $P<0.01)$.

Conclusions: It is shown that corilagin is a potential component to relieve cholestasis through inflammationrelated and oxidation-related pathway.

\section{Background}

Cholestasis is a reduction in bile flow that leads to the intrahepatic accumulation of bile acids and other toxic compounds with progression of liver pathology, including hepatocellular injury and fibrosis [1]. Recent studies have demonstrated that inflammatory injuries and oxidative stress occur in the liver with cholestasis [2]. Inflammatory stimulators induce signaling pathways within hepatocytes either directly, or through activation of proinflammatory cytokines, which result in suppressed expression and function of key hepatobiliary transporters and repressed expression and activity of a large number of nuclear transcriptional regulators, subsequently leading to rapid and profound reductions in bile flow [3].

\footnotetext{
* Correspondence: chinesemd@hotmail.com

${ }^{\dagger}$ Equal contributors

${ }^{4}$ Department of Infectious Disease and Hepatology, Union Hospital, Tongji Medical College, Huazhong University of Science and Technology, Wuhan 430022, PR China

Full list of author information is available at the end of the article
}

This procedure enrolls neutrophils to accumulate in the liver that evoke reactive oxygen species (ROS) to produce oxidative stress and liver injury [4].

Generally in clinical practice, treatments for cholestasis remain largely nonspecific and often ineffective [5]. UDCA (ursodeoxycholic acid) is the therapeutic agent most widely used for the treatment of cholestatic hepatopathies [6]. Recent research indicated that UDCA administration early after orthotopic liver transplantation improved serum liver tests and decreased the incidence of biliary sludge and cast within the 1st postoperative year [7]. But it was concerned that further studies should be needed evaluating a longer administration of UDCA that might be even more beneficial [8]. Further, in order to obtain an effect in acute cholestasis in non-surgery condition, such as acute hepatitis, hepatic failure or drug-induced hepatic injury, UDCA should be combined with corticosteroids [9], which indicated that UDCA was a limited choice in those diseases. Another effective is glucocorticoids. It was reported that 
dexamethasone can decrease cholestatic liver injury within hours after bile duct ligation, which can enhance the mitochondrial biogenesis and modulate the intrinsic pathway of apoptosis following bile duct ligation [10]. But the side effects of glucocorticoids limit use in many infection or bleeding-associated diseases.

Corilagin, a member of the tannin family with its molecular formula $\mathrm{C}_{27} \mathrm{H}_{22} \mathrm{O}_{18}$ [11], has been discovered in many medicinal plants such as Phyllanthus speices etc. [12]. Recent research indicated that corilagin has multiple activities including antioxidative, antiinflammatory, antiapoptotic, hepatoprotective and others. It was reported that corilagin could attenuate tert-butyl hydroperoxide-induced oxidative stress injury in microglial cells, which suggests that corilagin should be a potential candidate for the treatment of oxidative stress-induced neurodegenerative diseases [13]. It has been shown that corilagin has the potential to reduce HSV-1-induced inflammatory insult to the brain [14] and an anti-inflammatory activity in a cellular model [15]. Furthermore, it was confirmed that corilagin is an inhibitor of TNF- $\alpha$ [16] and can restrain radiation-induced microglia activation via suppression of the NF-kB pathway [17], and corilagin is protective against GalN/LPS-induced liver injury through suppression of oxidative stress and apoptosis [18]. Our recent research showed that corilagin can alleviate the hepatic fibrosis caused by egg granuloma in Schistosoma japonicum infection [19].

As nowadays there are no specific remedies for cholestasis, while corilagin can alleviate the impairment caused by inflammation and oxidation, we chose corilagin to treat the animal model of acute cholestasis in order to define the activity to interfere with inflammation-related and oxidative stress pathway in cholestasis pathogenesis.

\section{Methods}

\section{Chemicals and reagents}

All chemicals were purchased from Gibco (Invitrogen, city, country) or HyClone (Thermo Scientific, city, country) (like PBS and other basic stuff - this was an explanation for the authors and should not appear in the text) unless indicated otherwise. Affinity-purified rabbit anti-rat NF-kB p65 was received from Santa Cruz Biotechnology (Santa Cruz, CA). Biotin-conjugated goat anti rabbit IgG and streptavidin-horseradish peroxidase (HRP) conjugate were obtained from Kangcheng Biotech Company (Shanghai, China). Corilagin was provided by Dr. Jun-Yan Tao and friendly offered by Prof. Ji-Kai Liu, Kunming Institute of Botany, Chinese Academy of Science.

\section{Animals}

Male Sprague-Dawley rats weighing 200-220g were purchased from the Experimental Animal Center of Tongji Medical Colllege, Huazhong University of Science and Technology. The rats were maintained under standard laboratory conditions at a temperature of $25 \pm 2^{\circ} \mathrm{C}$, a relative humidity of $50 \pm 15 \%$ and normal circadian rhythm (12-h dark/12-h light). The animals were fed normal diet and water ad libitum. All study protocols were approved by internationally accepted principles and the Guidelines for the Care and Use of Laboratory Animals of Huazhong University of Science and Technology.

\section{Model and control establishment}

90 rats were equally divided into 5 groups i.e. corilagin, UDCA, dexamethasone, model and blank control groups. Corilagin was prepared as $1.6 \%$ suspension; UDCA (Dr. Falk Pharma GmbH, Freiburg, Germany) was prepared as $0.6 \%$ suspension with water; dexamethasone (Zhejiang XianJu Pharmaceutical Company Ltd., Zhejiang, China) was dissolved in water at a concentration of $0.045 \%$; ANIT (Sigma, St. Louis, MO) was dissolved in Sesame Oil at a concentration of $1 \%$. Before establishing the animal model, corilagin $(40 \mathrm{mg} / \mathrm{kg} / \mathrm{d})$, UDCA $(60 \mathrm{mg} / \mathrm{kg} / \mathrm{d})$ and dexamethasone $(1.8 \mathrm{mg} / \mathrm{kg} / \mathrm{d})$ were intragastrically administrated to the rats in respective group for 4 days. Model and blank control were fed by normal saline. All groups did not stop being administrated treating agent daily until executed. At the 5th day after administration and fasting for $12 \mathrm{~h}$, all group except normal control were intragastrically administrated ANIT $(50 \mathrm{mg} / \mathrm{kg}$ ) for modeling. At the same day, with $8 \mathrm{~h}$ interval the rats were still fed by respective drug or control agent. At $24 \mathrm{~h}, 48 \mathrm{~h}, 72 \mathrm{~h}$ after modeling, every 6 rats in each group were executed for taking specimens. The living conditions of the victims were observed as our previous study [20].

\section{Specimen collection}

The procedure abided by our past experiment [21]. Following anesthesia with $6 \%$ chloral hydrate by intraperitoneal injection, the rat's abdomen was opened and abdominal aorta was separated. At the same time the common bile duct was intubated for draining bile. Then $3 \mathrm{ml}$ arterial blood was collected in coagulant test tube. Blood serum was obtained after $3250 \mathrm{~g}$ centrifugation and stored at $-20^{\circ} \mathrm{C}$ until testing. Subsequently, rat's liver was cut by aseptic, RNase-free apparatus. After washing with normal saline, the whole hepatic tissue was divided into two parts: one was sheared and stored at $-80^{\circ} \mathrm{C}$, the other was fixed in $10 \%$ formalin for $48 \mathrm{~h}$ and then dehydrated, followed by imbedding in paraffin and slicing.

\section{Serum markers of liver damage}

The serumal total bilirubin, direct bilirubin, alanine aminotransferase (ALT) and aspartate aminotransferase (AST) were assayed by Aeroset Fully-auto Chemistry Analyzer provided by Abbott Co LTD. 


\section{Immunohistochemistry assay}

Streptavidin-perosidase (SP) immunohistochemical assay was employed to detect expression of the nuclear translocation of NF-kB. The slides of hepatic tissue were soaked in 3\% $\mathrm{H} 2 \mathrm{O} 2$-methanol solution for $20 \mathrm{~min}$ in order to block endogenous peroxydase. In the next $1 \%$ Triton X-100 was added at $37^{\circ} \mathrm{C}$ for $5 \mathrm{~min}$, followed by washing with PBS. After incubation with normal goat serum at room temperature for $20 \mathrm{~min}$, rabbit anti-rat NF- $\mathrm{kB}$ p65 IgG antibody (1:200) was added dropwise and the slides were stored at $4^{\circ} \mathrm{C}$ overnight. The next day slides were washed with PBS and incubated with biotin-conjugated goat anti rabbit IgG for $30 \mathrm{~min}$ at $37^{\circ} \mathrm{C}$. After another washing with PBS streptavidin-HRP was added and incubated for $30 \mathrm{~min}$ at $37^{\circ} \mathrm{C}$. The slides were thoroughly washed with PBS 3 times for $5 \mathrm{~min}$ and stained with 3,3'-diaminobenzidine. Following normal dehydration, lucidification and mounting the slides were analysed under microscope (Olympus, Tokyo, Japan) as specified in our previous studies [22-25] and digital images were captured with camera (Olympus, Tokyo, Japan).

\section{$\mathrm{NO}$ and oxidates assay}

Myeloperoxidase (MPO), malondialdehyde (MDA), superoxide dismutase (SOD), and nitric oxide (NO) were quantified by the respective assay kits (Nanjing Jiancheng Bioengineering Institute, Nanjing, China) according to the instructions of the manufacturer. The procedures were also described in our previous studies [26,27].

\section{Purity determination of corilagin by HPLC}

Purity of corilagin was determined by HPLC. The procedure abided by our previous study [28,29]. Briefly, a Hanbon-Kromasil $5 \mu \mathrm{m} \mathrm{C18}$ column was used at $30^{\circ} \mathrm{C}$ with the wavelength of $268 \mathrm{~nm}$ for detection. The mobile phase was composed of $0.5 \%$ phosphoric acid and methyl cyanide with a ratio of $76: 24$ and the injection volume was $10 \mu$ l. Corilagin standard substance (purity $>99 \%$ ) was offered by China National Institute for the Control of Pharmaceutical and Biological Products. The purity of corilagin was calculated to $62.14 \%$. (Figure 1)

\section{Statistical analysis}

Data were presented as mean \pm S.D. Comparisons of measurement data between multiple groups were performed with one-way ANOVA test. Comparison of the positive rate of NF- $\mathrm{KBp} 65$ between different groups was carried out by Pearson chi-square tests. Statistical significance was considered significant when $\mathrm{P}<0.05$. Whole process was performed with SPSS 12.0 software.

\section{Results}

\section{Living condition of the models}

In normal group, the rats had clear urine and velvet and glossy hair. The bile could be easily drained. When taken out, the liver tissue was ruddy and lubricous. As comparison, it could be observed that the rats in model group were inactive and anorectic. Their hair was in a mess and urine was deep yellow. The drained bile decreased and their liver lost ruddy and lubricous appearance with a few of nodules at surface. The intestinal canal of the model was distended or even of toxic meteorism. Compared to model group, the rats in corilagin group appeared more active and had more appetite, more clear urine. The liver showed more ruddy and lubricous appearance with fewer nodules at surface with more easily drained bile, and the intestinal canal was less distended. The rats in UDCA group showed similar effect as corilagin group. The rats in dexamethasone group showed inactive state with proceeding weight loss and mess hair. The color of urine was clearer than that in model group, while hemorrhagic points emerged at mucous membrane in eyes, nose, month and surface of the liver. The bile in dexamethasone group could not be drained easily and the intestinal canal was dilated like that of model group.

\section{Serum markers of liver damage}

As shown in Figure 2, when compared with model control, corilagin had a significantly decreasing effect on total bilirubin and direct bilirubin $(\mathrm{P}<0.01)$. At $24 \mathrm{~h}$, the effect of corilagin on total bilirubin and direct bilirubin was superior to that of UDCA but inferior to that of dexamethasone, while at $48 \mathrm{~h}$ corilagin was the most effective agent $(\mathrm{P}<0.01)$ but at $72 \mathrm{~h}$ the three agents had notable effect on total bilirubin and direct bilirubin level $(\mathrm{P}<0.01)$. Corilagin had no effect on serum aminotransferase release. UDCA showed an effect on $72 \mathrm{~h}$ and dexamethasone was found to have an effect on $24 \mathrm{~h}$ and $72 \mathrm{~h}$ on total bilirubin and direct bilirubin level $(\mathrm{P}<0.01)$.

\section{Pathological manifestation of hepatic tissue}

As shown in Figure 3, pathological features were differently presented in each group. In normal group, hepatic tissue showed intact hepatic lobules, orderly liver cell cord, hepatic cell with uniform stain, epithelial cells of bile duct without damage, and no infiltration of neutrophilic granulocyte. In model group, the liver tissue showed typical pathological changes. At $24 \mathrm{~h}$, destruction of hepatic lobules, hydropic degeneration or feather-like degeneration in liver cells, swelling amotic bile duct epithelial cell and infiltration of neutrophilic granulocyte in portal area were observed. At $48 \mathrm{~h}$, the liver cells showed more significantly swelling, cytoplasm with puff, ununiformed nucleus with accumulative chromatin and 


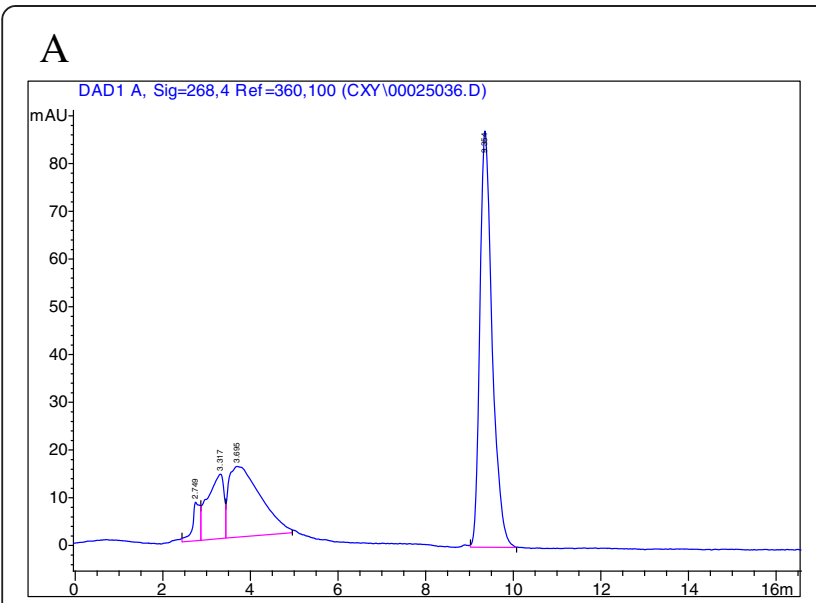

B

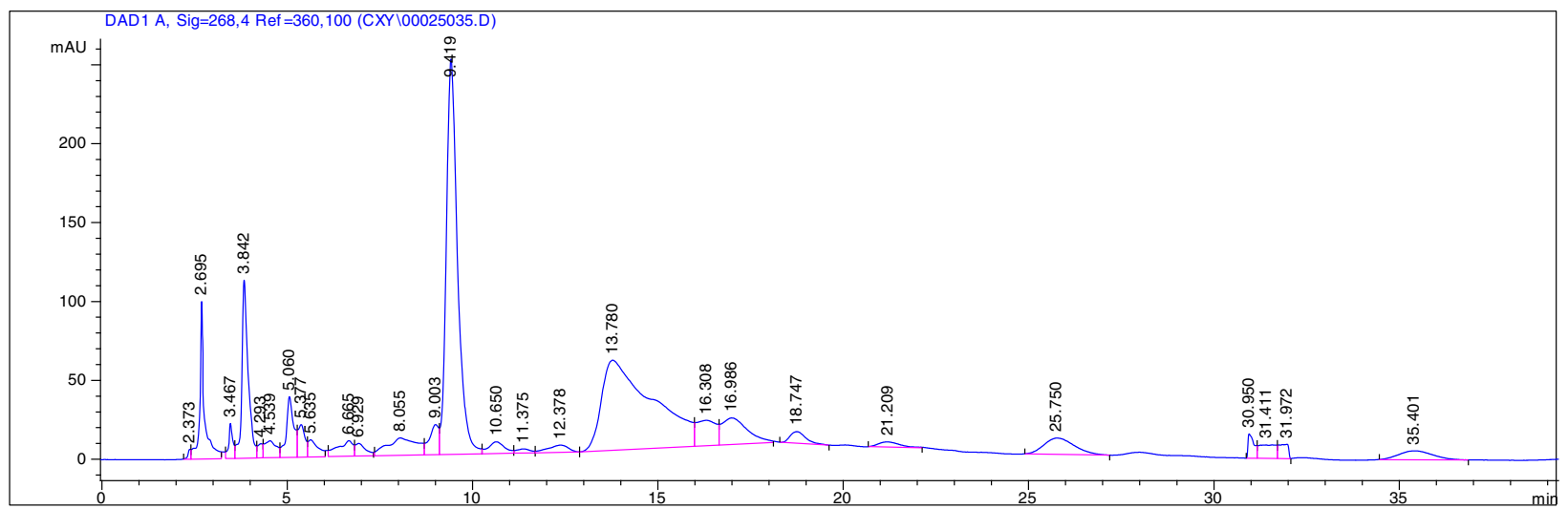

Figure 1 Purity determination of corilagin by high pressure/performance liquid chromatography (HPLC). A: The HPLC curve of corilagin standard substance; $\mathbf{B}$ : The HPLC curve of corilagin sample. Purity of corilagin was determined by HPLC. A Hanbon-Kromasil $5 \mu m$ C18 column was used at $30^{\circ} \mathrm{C}$ with the wavelength of $268 \mathrm{~nm}$ for detection. The mobile phase was composed of $0.5 \%$ phosphoric acid and methyl cyanide with a ratio of $76: 24$ and the injection volume was $10 \mu$. The purity of corilagin was calculated to $62.14 \%$.

enlarged, strong-stained nucleolus. The liver tissue showed plenty of punctiform or focused necrotic zones and proliferation of Kupffer cells and bile duct epithelial cells all over the visual field. The bile duct exhibited a constrictive canal with necrotic cells and bile thrombus. Many necrotic hepatocytes and infiltrated neutrophils were present around the bile duct. At $72 \mathrm{~h}$, the pathological changes were recovered slightly but still featured with necrotic regions and neutrophil infiltration. In the corilagin group the pathological changes were significantly lower than in the model group. The manifestations in the UDCA group were a little severer than in the corilagin group while in the dexamethasone group the pathological impairment appeared more aggravated.

\section{Expression of NF-KBp65 examined by immunohistochemistry}

As shown in Figure 4, it was shown that in normal group the staining of NF-kBp65 in nucleus was not remarkable. After ANIT administration, the positive rate of NF-kBp65 staining in nucleus significantly rose $(\mathrm{P}<0.01)$. With corilagin treatment, the rate of NF-kBp65 staining in nucleus was decreased markedly $(\mathrm{P}<0.01)$. In UDCA and dexamethasone group the rate was also significantly lower than that in model group but higher than that in corilagin group $(\mathrm{P}<0.05)$.

\section{Levels of MPO, MDA, SOD and NO in tissue}

As shown in Figure 5, the level of MPO and MDA significantly rose in the model group $(\mathrm{P}<0.01)$. At all-time points the levels of the two enzymes were notably downregulated by corilagin treatment and even lower than the normal level. As control, UDCA had no similar effect as corilagin, and dexamethasone only had an effect on MDA at $72 \mathrm{~h}(\mathrm{P}<0.05)$. In contrast, the levels of SOD and $\mathrm{NO}$ in tissue decreased notably after ANIT stimulation $(\mathrm{P}<0.05$ or $\mathrm{P}<0.01$, respectively), while in the corilagin group the levels of SOD and NO were remarkably elevated $(\mathrm{P}<0.05$ or $\mathrm{P}<0.01$, respectively). UDCA had not the same effects except for SOD at $48 \mathrm{~h}$. 

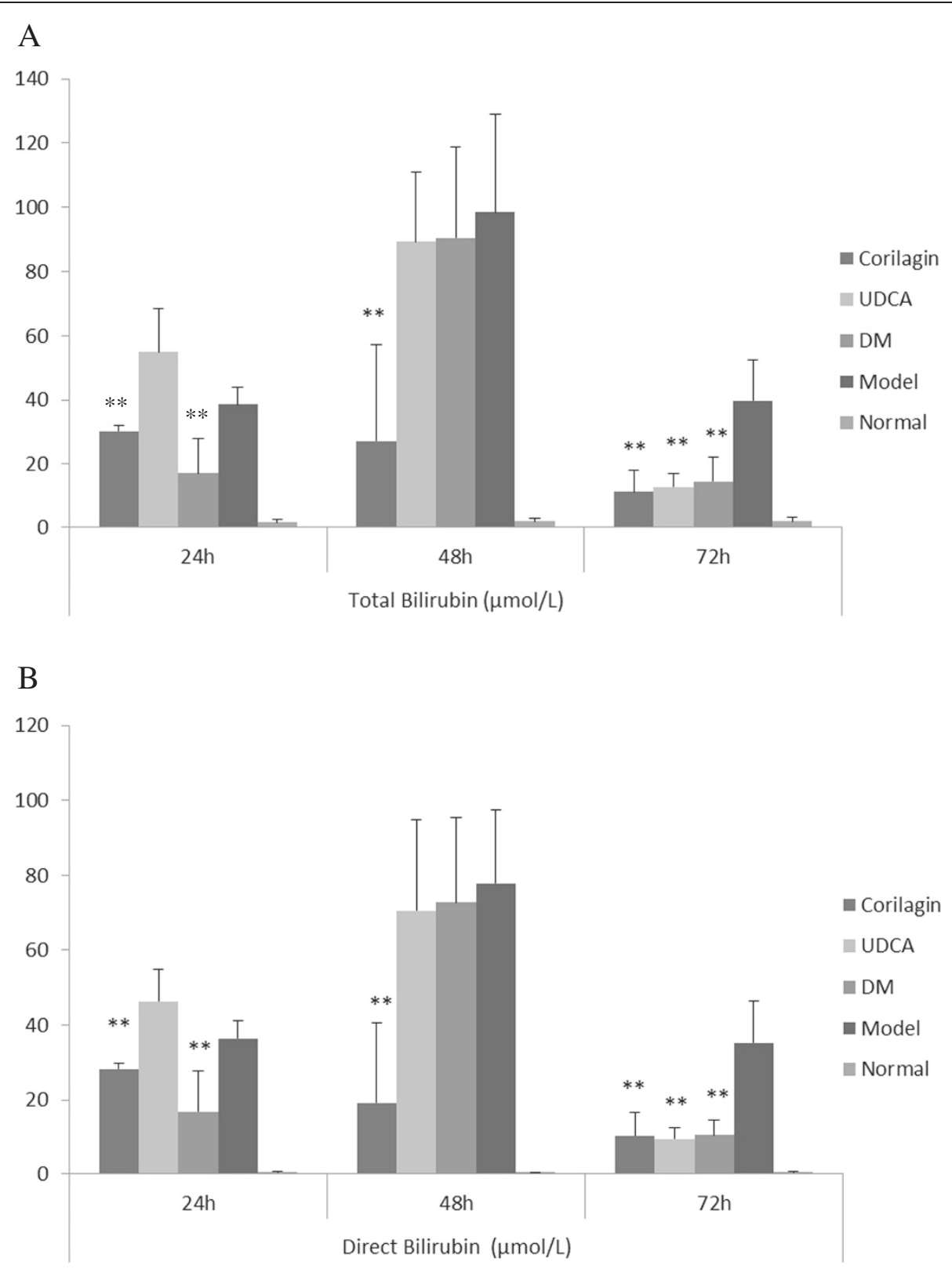

Figure 2 Effect of corilagin on Liver Functional Test at $\mathbf{2 4 h}$, 48h, $\mathbf{7 2 h}$. A: On Total Bilirubin; B: On Direct Bilirubin. The data were shown as mean \pm SD; ${ }^{*} p<0.05$ compared to model group; ${ }^{* *} p<0.01$ compared to model group. When compared with model control, corilagin had a significantly decreasing effect on total bilirubin and direct bilirubin $(P<0.01)$. At $24 \mathrm{~h}$, the effect of corilagin on total bilirubin and direct bilirubin was superior to that of UDCA but inferior to that of dexamethasone, while at $48 \mathrm{~h}$ corilagin was the most effective agent $(P<0.01)$ but at $72 \mathrm{~h}$ the three agents had notable effect on total bilirubin and direct bilirubin level $(P<0.01)$. UDCA showed an effect on $72 \mathrm{~h}$ and dexamethasone was found to have an effect on $24 \mathrm{~h}$ and $72 \mathrm{~h}$ on total bilirubin and direct bilirubin level $(P<0.01)$.

Dexamethasone had an up-regulating effect on SOD at $48 \mathrm{~h}$, and on $\mathrm{NO}$ at $24 \mathrm{~h}$ and $72 \mathrm{~h}(\mathrm{P}<0.05$ or $\mathrm{P}<0.01$, respectively).

\section{Discussion}

Cholestasis is characterized by impaired bile flow, reduction of bile acids in the intestine, and retention of bile acids in the liver. Rats taken in alpha naphthyliso- thiocyanate (ANIT) have been one of the most common experimental models of intrahepatic cholestasis and used extensively, which was permitted to describe not only cholestatic alterations but also compensatory mechanisms [30]. The liver in ANIT-treated rats showed cholangiolitic hepatitis characterized by intrahepatic cholestasis, necrosis of hepatocytes and biliary epithelial cells and bile obstruction [31]. 

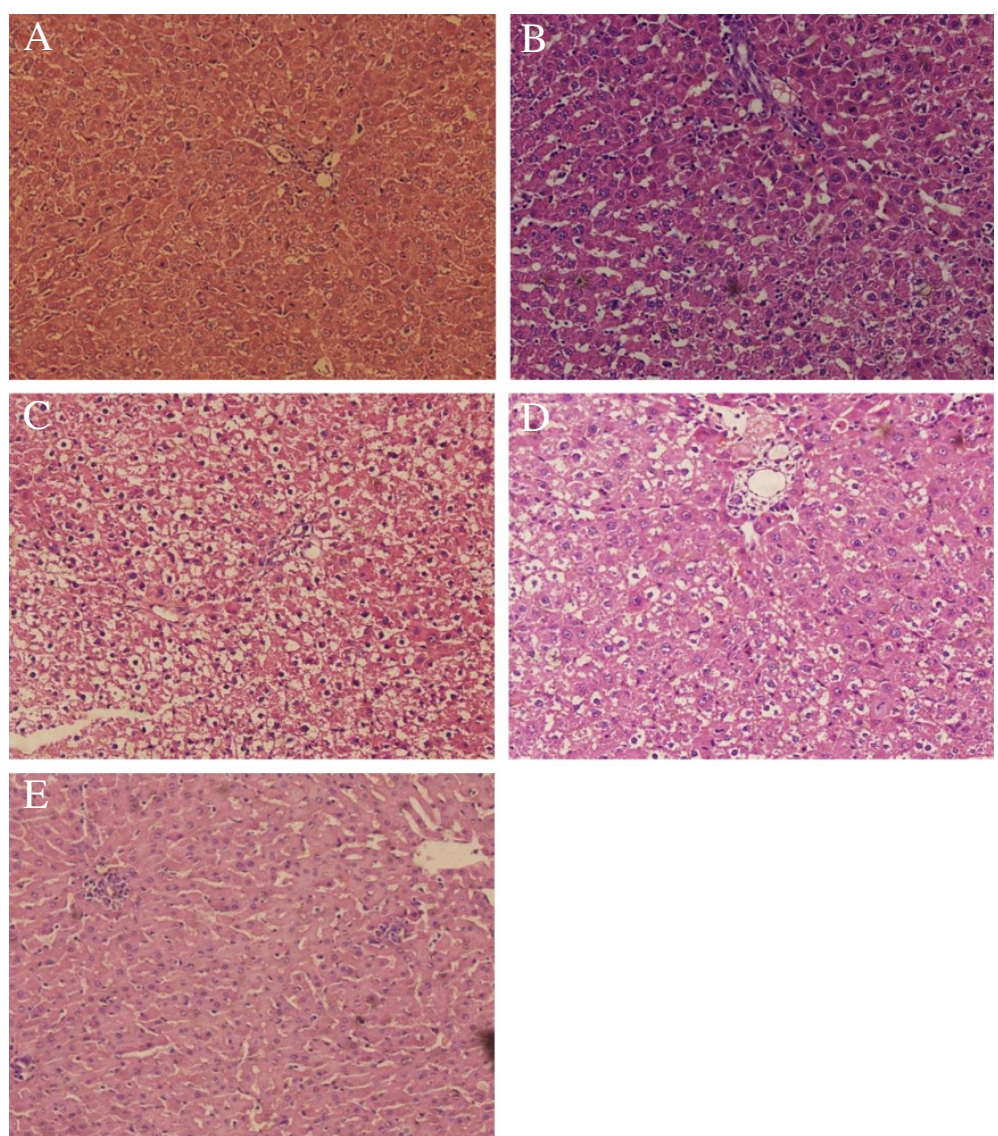

Figure 3 Effect of corilagin on pathological manifestation of hepatic tissue at $\mathbf{4 8 h}$. A: Corilagin group; B: UDCA group; C: Dexamethasone group; D: Model group; E: Normal group. In normal group, hepatic tissue showed intact hepatic lobules, orderly liver cell cord, hepatic cell with uniform stain, epithelial cells of bile duct without damage, and no infiltration of neutrophilic granulocyte. In model group, the liver tissue showed typical pathological changes. At 48h, the liver cells showed more significantly swelling, cytoplasm with puff, ununiformed nucleus with accumulative chromatin and enlarged, strong-stained nucleolus. The liver tissue showed plenty of punctiform or focused necrotic zones and proliferation of Kupffer cells and bile duct epithelial cells all over the visual field. The bile duct exhibited a constrictive canal with necrotic cells and bile thrombus. Many necrotic hepatocytes and infiltrated neutrophils were present around the bile duct. In the corilagin group the pathological changes were significantly lower than in the model group. The manifestations in the UDCA group were a little severer than in the corilagin group while in the dexamethasone group the pathological impairment appeared more aggravated.

In current clinical practice, initial assessment of hepatobiliary diseases is accomplished by measuring serum concentrations of bile acids and bilirubin as well as serum activities of liver-associated enzymes which reveal information about the state of liver. Our previous study showed that the indexes of liver damage and pathological changes start to rise at $24 \mathrm{~h}$ after ANIT treatment, reach a maximum at $48 \mathrm{~h}$ and trend to restore at $72 \mathrm{~h} \mathrm{[21].} \mathrm{The} \mathrm{data} \mathrm{of} \mathrm{the} \mathrm{present} \mathrm{study} \mathrm{imply} \mathrm{that} \mathrm{the}$ most effective change after corilagin treatment refers to bilirubin, both total and direct. Compared to UDCA and dexamethasone, corilagin showed a protective effect on the elevation of serum bilirubin after hepatic injury. However, there is no obvious evidence of corilagin to alleviate aminotransferases, which indicates that corilagin has a stronger effect on relieving bile or bile duct disorders.
The ANIT hepatotoxicity is attributed to both glutathione and blood neutrophils. Glutathione can form a reversible S-conjugate with ANIT that is critical in shuttling ANIT into bile, where it induces a group of toxic substances[32], and neutrophils may be activated during ANIT exposure to release cytotoxic proteases that cause injury to target cells [33]. In our study, with ANIT treatment the rat liver showed typical damage of neutrophilic infiltration, necrosis of hepatocytes, proliferation of inflammatory cells and epithelial cells of bile duct and formation of bile thrombus. By corilagin intervention, it could be observed that improvement of the acute hepatic impairment was achieved, which shows the protective effect of corilagin on hepatic pathology.

ANIT-evoked hepatotoxins cause severe neutrophilic inflammation around portal tracts and bile ducts and induce significant inflammatory reactions in the liver, 

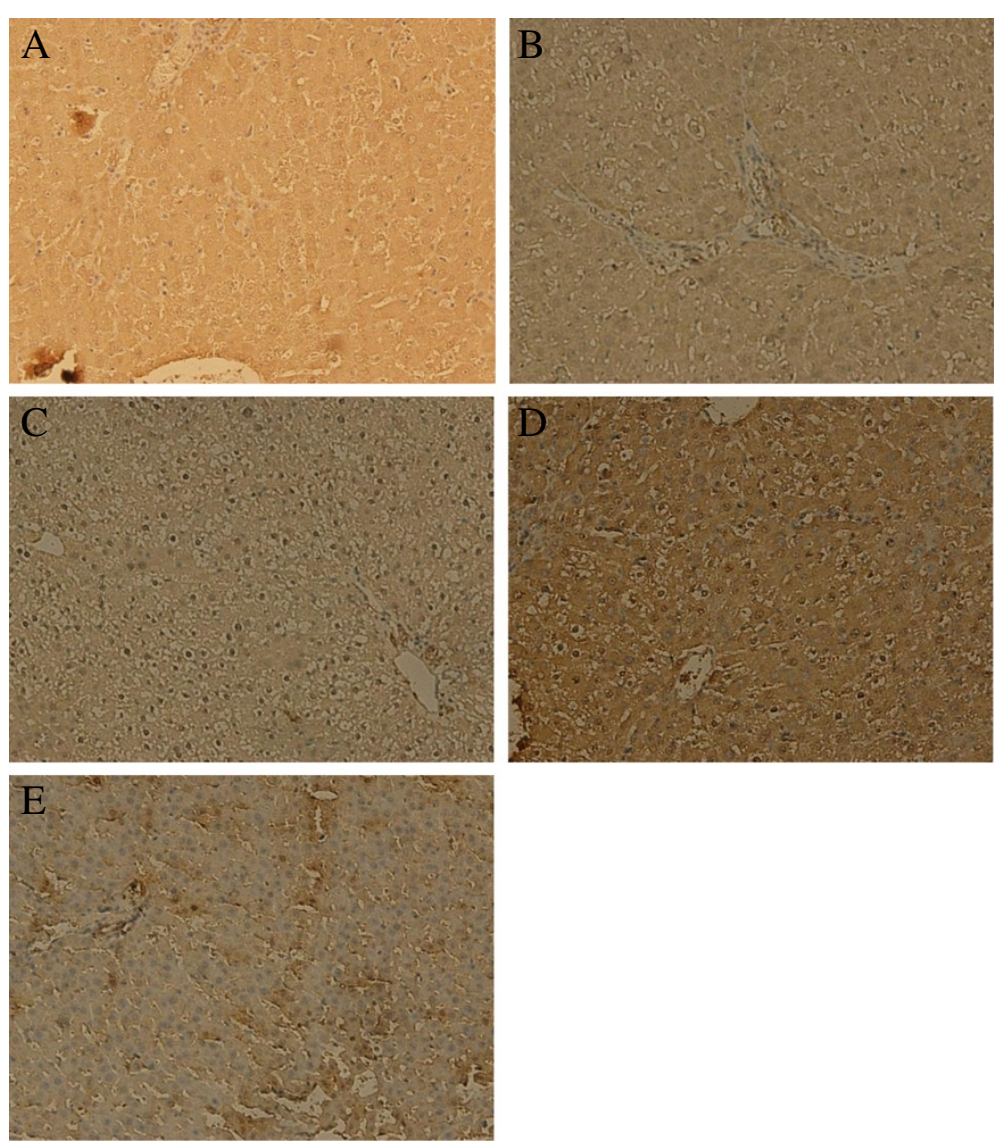

Figure 4 Effect of corilagin on NF-KBp65 expression at 48h. The activated NF-KBp65 presented brown stain in nuclear and inactivated NF-KBp65 presented brown in cytoplasm. A: Corilagin group; B: UDCA group; C: Dexamethasone group. D: Model group; E: Normal group. It was shown that in normal group the staining of NF-KBp65 in nucleus was not remarkable. After ANIT administration, the positive rate of NF-kBp65 staining in nucleus significantly rose $(\mathrm{P}<0.01)$. With corilagin treatment, the rate of NF-KBp65 staining in nucleus was decreased markedly $(P<0.01)$. In UDCA and dexamethasone group the rate was also significantly lower than that in model group but higher than that in corilagin group $(P<0.05)$.

which suggests that the inflammation plays a central role in cholestatic hepatitis. Nuclear factor-kappa B (NF-kB) is a pivotal factor that transfers inflammatory signals from cytoplasm into the nucleus and induces a series of inflammatory responses in the cell $[34,35]$. In our present study, it was found that NF-kB was notably suppressed in the corilagin group, which demonstrates that corilagin has the efficacy to control inflammatory injuries during cholestasis in the liver.

The reactive oxygen species are generated by aerobic metabolism and environmental stressors. They can chemically modify proteins and alter their biological functions and, if the repair processes fail, oxidized proteins may become cytotoxic [36]. Myeloperoxidase (MPO), a heme-containing peroxidase abundantly expressed in neutrophils and monocytes, while produces the powerful oxidant hypochlorous acid and is a key contributor to the oxygen-dependent microbicidal activity of phagocytes, is linked to tissue damage in many diseases when it is excessive to generate MPO-derived oxidants [37]. In our research, corilagin displayed a strong effect on MPO, demonstrating the oxidative impairment of neutrophils can be prevented by corilagin. Malondialdehyde (MDA) is the principal and most studied product of polyunsaturated fatty acid peroxidation. So far, oxidative stress have been assessed by measuring the level of antioxidants or the concentration of substances derived from the action of oxygen free radicals on biological molecules, and measurement of MDA is considered an effective marker of oxidative stress in a biological sample [38]. In our study, it could be showed that corilagin has a significant effect on MDA expression, which implies corilagin has a strong potential to suppress oxidative stress when cholestasis occurs. After oxidative stress occurs, products of lipid peroxidation created in different biochemical reactions are normally removed by antioxidants, the compounds that are involved in effective scavenging of free radicals and in 

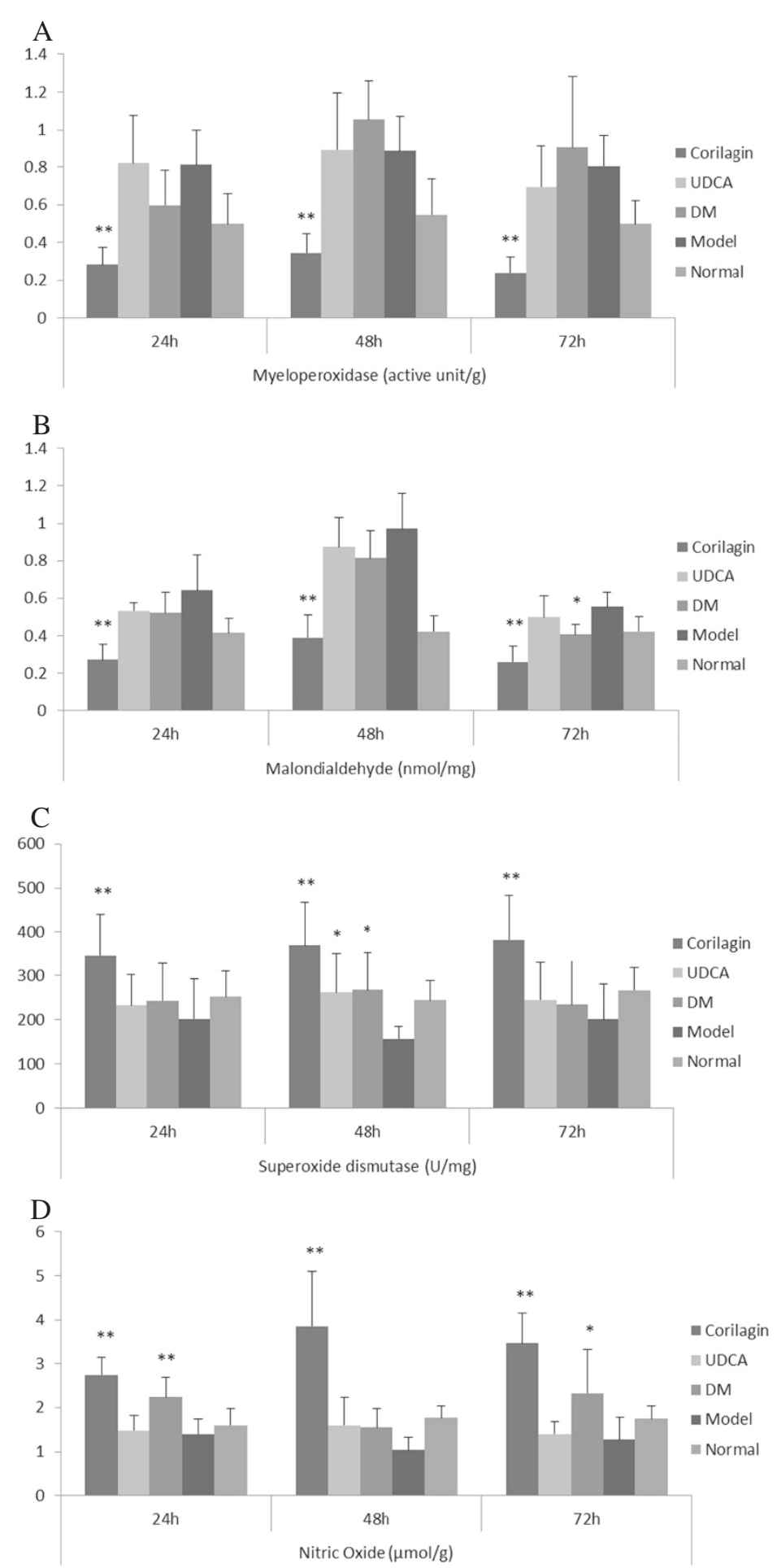

Figure 5 Effect of corilagin on anti-oxidative and nitric oxide at $24 \mathrm{~h}, \mathbf{4 8 h}, \mathbf{7 2 h}$. A: On myeloperoxidase; B: On malondialdehyde; C: On superoxide dismutase; $\mathbf{D}$ : On nitric oxide. The data were shown as mean $\pm \mathrm{SD} ;{ }^{*} p<0.05$ compared to model group; ${ }^{* *} p<0.01$ compared to model group. The level of MPO and MDA significantly rose in the model group $(P<0.01)$. At all-time points the levels of the two enzymes were notably down-regulated by corilagin treatment and even lower than the normal level. As control, UDCA had no similar effect as corilagin, and dexamethasone only had an effect on MDA at 72h $(P<0.05)$. In contrast, the levels of SOD and NO in tissue decreased notably after ANIT stimulation ( $P<0.05$ or $P<0.01$, respectively), while in the corilagin group the levels of $S O D$ and $N O$ were remarkably elevated $(P<0.05$ or $P<0.01$, respectively). UDCA had not the same effects except for SOD at 48h. Dexamethasone had an up-regulating effect on SOD at 48h, and on NO at 24h and $72 h(P<0.05$ or $\mathrm{P}<0.01$, respectively). 
suppressing the actions of reactive oxygen substances [39]. The most important enzymatic antioxidants are superoxide dismutase (SOD), a sort of enzyme that catalyze the dismutation of superoxide radical into hydrogen peroxide $(\mathrm{H} 2 \mathrm{O} 2)$ and molecular oxygen $(\mathrm{O} 2)$ and consequently present an important defense mechanism against superoxide radical toxicity [40]. In the present research, the SOD level in corilagin group was markedly higher than that in model group, which showed the anti-oxidative effect of corilagin.

Nitric oxide (NO) plays a critical role on hepatic metabolism whether liver is in normal condition or in injury by different agents. Although some experiments show controversial effect of NO in the liver on pathological condition, literature from the past 15 years seems to reinforce the consensus that $\mathrm{NO}$ is indeed protective. Some of the protective actions of NO are due to its potential as an antioxidant and anti-inflammatory agent, along with its beneficial effects on cell signaling and inhibition of nuclear proteins, such as NF-kappa B and AP-1, especially when ischemia occurs [41,42]. The supplementation with a NO donor prevented caspase-3 activity and apoptosis induced by bile acids in cultured rat hepatocytes [43]. In our experiment, after corilagin treatment the expression of NO rose notably, which implies corilagin can improve circulation in the liver.

Corilagin has been found in many medicinal plants and some advanced technologies have been employed to detect the content of corilagin in those plants [44]. Although corilagin can be synthesized by chemicals, the actual output for synthesis is limited [11]. Thereby some novel techniques were used to purify corilagin from plants [45]. In our research, we identified the content of corilagin in extraction and the purity of corilagin is dominant in the extraction.

\section{Conclusions}

In conclusion, we found that corilagin has the capability to ensure hepatic protection, to block NF- $\mathrm{KB}$ pathway, to provide anti-oxidative effects and to improve hepatic circulation in experimental intrahepatic cholestasis, which suggests that corilagin is a putative medication to treat cholestasis. It is shown that corilagin has the potential to cure inflammation-related and oxidationrelated diseases. The further proceeding might be to investigate how corilagin interacts with the inflammatory and oxidative signal pathways in cell and animal models.

\section{Competing interests}

The authors declare that they have no competing interests.

\section{Authors' contributions}

FJ participated in the serum markers of liver damage and drafted the manuscript. DC took part in immunohistochemistry. JY Tao carried out purity determination of corilagin. RP and YGparticipated in detection of oxidationrelated enzymes. PY performed animal feeding and observation. JD and SZ participated in the design of the study and performed the statistical analysis. LZ conceived of the study, and participated in its design and coordination. All authors read and approved the final manuscript.

\section{Acknowledgement}

This study is sponsored by China National Natural Science Fund No. 30901248/H1906 and No. 81071779/H1607.

\section{Author details}

${ }^{1}$ Department of Neurosurgery, Neuro-oncology Laboratory, Affiliated Hospital of Jining Medical College, Jining, Shandong 272029, PR China. ${ }^{2}$ Liver Disease Center, Department of Infectious Disease, Second Xiangya Hospital, Xiangya Medical School, Central South University, Changsha 410011, PR China. ${ }^{3}$ Department of Bioengineering and Therapeutic Sciences, School of Pharmacy, UCSF, CA 94143, USA. ${ }^{4}$ Department of Infectious Disease and Hepatology, Union Hospital, Tongji Medical College, Huazhong University of Science and Technology, Wuhan 430022, PR China. ${ }^{5}$ Department of Neurology, Union Hospital, Tongji Medical College, Huazhong University of Science and Technology, Wuhan 430022, P.R. China. ${ }^{6}$ Central Lab, Union Hospital, Tongji Medical College, Huazhong University of Science and Technology, Wuhan 430022, P.R. China.

Received: 11 November 2012 Accepted: 26 April 2013

Published: 3 May 2013

\section{References}

1. Crocenzi FA, Zucchetti AE, Boaglio AC, Barosso IR, Sanchez Pozzi EJ, Mottino $A D$, Roma MG: Localization status of hepatocellular transporters in cholestasis. Front Biosci 2012, 17:1201-1218.

2. Jaeschke $\mathrm{H}$ : Reactive oxygen and mechanisms of inflammatory liver injury: Present concepts. J Gastroenterol Hepatol 2011, 26:173-179.

3. Kosters A, Karpen SJ: The role of inflammation in cholestasis: clinical and basic aspects. Semin Liver Dis 2010, 30:186-194.

4. Copple BL, Jaeschke $H$, Klaassen CD: Oxidative stress and the pathogenesis of cholestasis. Semin Liver Dis 2010, 30:195-204.

5. Hirschfield GM, Heathcote EJ: Cholestasis and cholestatic syndromes. Curr Opin Gastroenterol 2009, 25:175-179.

6. Roma MG, Toledo FD, Boaglio AC, Basiglio CL, Crocenzi FA, Sánchez Pozzi EJ: Ursodeoxycholic acid in cholestasis: linking action mechanisms to therapeutic applications. Clin Sci (Lond) 2011, 121:523-544.

7. Wang SY, Tang HM, Chen GQ, Xu JM, Zhong L, Wang ZW, Deng GL, Xing TH, Lu LG, Peng ZH: Effect of ursodeoxycholic acid administration after liver transplantation on serum liver tests and biliary complications: a randomized clinical trial. Digestion 2012, 86:208-217.

8. Herzer K, Fingas CD, Canbay A: Does ursodeoxycholic acid exert a protective effect on liver grafts in orthotopic liver transplantation? Digestion 2012, 86:206-207.

9. Wree A, Dechêne A, Herzer K, Hilgard P, Syn WK, Gerken G, Canbay A: Steroid and ursodesoxycholic Acid combination therapy in severe drug-induced liver injury. Digestion 2011, 84:54-59.

10. Tiao MM, Lin TK, Chen JB, Liou CW, Wang PW, Huang CC, Chou YM, Huang $\mathrm{YH}$, Chuang JH: Dexamethasone decreases cholestatic liver injury via inhibition of intrinsic pathway with simultaneous enhancement of mitochondrial biogenesis. Steroids 2011, 76:660-666.

11. Yamada H, Nagao K, Dokei K, Kasai Y, Michihata N: Total synthesis of (-)-Corilagin. J Am Chem Soc 2008, 130:7566-7567.

12. Shen ZQ, Dong ZJ, Peng H, Liu JK: Modulation of PAI-1 and tPA activity and thrombolytic effects of Corilagin. Planta Med 2003, 69:1109-1112.

13. Chen $Y$, Chen $C$ : Corilagin prevents tert-butyl hydroperoxide-induced oxidative stress injury in cultured N9 murine microglia cells. Neurochem Int 2011, 59:290-296.

14. Guo YJ, Zhao L, Li XF, Mei YW, Zhang SL, Tao JY, Zhou Y, Dong JH: Effect of Corilagin on anti-inflammation in HSV-1 encephalitis and HSV-1 infected microglias. Eur J Pharmacol 2010, 635:79-86.

15. Zhao L, Zhang SL, Tao JY, Pang R, Jin F, Guo YJ, Dong JH, Ye P, Zhao HY, Zheng GH: Preliminary exploration on anti-inflammatory mechanism of Corilagin (beta-1-O-galloyl-3,6-(R)-hexahydroxydiphenoyl-D-glucose) in vitro. Int Immunopharmacol 2008, 8:1059-1064.

16. Okabe S, Suganuma M, Imayoshi Y, Taniguchi S, Yoshida T, Fujiki H: New TNF-alpha releasing inhibitors, geraniin and Corilagin, in leaves of Acer nikoense, Megusurino-ki. Biol Pharm Bull 2001, 24:1145-1148. 
17. Dong XR, Luo M, Fan L, Zhang T, Liu L, Dong JH, Wu G: Corilagin inhibits the double strand break-triggered NF-kappaB pathway in irradiated microglial cells. Int J Mol Med 2010, 25:531-536.

18. Kinoshita S, Inoue Y, Nakama S, Ichiba T, Aniya Y: Antioxidant and hepatoprotective actions of medicinal herb, Terminalia catappa L. from Okinawa Island and its tannin Corilagin. Phytomedicine 2007, 14:755-762.

19. Huang YF, Zhang SL, Jin F, Cheng D, Zhou YP, Li HR, Tang ZM, Xue J, Cai W, Dong $\mathrm{JH}$, Zhao L: Activity of corilagin on post-parasiticide liver fibrosis in Schistosomiasis animal model. Int J Immunopathol Pharmacol 2013, 26:85-92.

20. Jin F, Gao C, Zhao L, Zhang H, Wang HT, Shao T, Zhang SL, Wei YJ, Jiang $X B$, Zhou YP, Zhao HY: Using CD133 positive U251 glioblastoma stem cells to establish nude mice model of transplanted tumor. Brain Res 2011, 1368:82-90

21. Ding $Y$, Zhao L, Mei H, Zhang SL, Huang ZH, Duan YY, Ye P: Exploration of Emodin to treat alpha-naphthylisothiocyanate-induced cholestatic hepatitis via anti-inflammatory pathway. Eur J Pharmacol 2008, 590:377-386.

22. Jin F, Zhao L, Guo YJ, Zhao WJ, Zhang H, Wang HT, Shao T, Zhang SL, Wei YJ, Feng J, Jiang XB, Zhao HY: Influence of Etoposide on anti-apoptotic and multidrug resistance-associated protein genes in CD133 positive U251 glioblastoma stem-like cells. Brain Res 2010, 1336:103-111.

23. Liu P, Zhao L, Zhang SL, Xiang JZ: Modified Wendan Decoction can Attenuate Neurotoxic Action Associated with Alzheimer's Disease. Evid Based Complement Alternat Med 2009, 6:325-330

24. Jin F, Zhao L, Zhao HY, Guo SG, Feng J, Jiang XB, Zhang SL, Wei YJ, Fu R, Zhao JS: Comparison between cells and cancer stem-like cells isolated from glioblastoma and astrocytoma on expression of anti-apoptotic and multidrug resistance-associated protein genes. Neuroscience 2008, 154:541-550.

25. Zhou YP, Zhang SL, Cheng D, Li HR, Tang ZM, Xue J, Cai W, Dong JH, Zhao $L$ : Preliminary exploration on anti-fibrosis effect of kaempferol in mice with Schistosoma japonicum infection. Eur J Inflamm 2013, 11:161-168

26. Li XF, Guo YJ, Zhang DM, Chen Z, Wei X, Li YH, Zhang SL, Tao JY, Dong JH, Mei YW, Li LL, Zhao L: Protective activity of the ethanol extract of Cynanchum paniculatum (BUNGE) Kitagawa on treating herpes simplex encephalitis. Int J Immunopathol Pharmacol 2012, 25:259-266.

27. Li XF, Guo YJ, Wang ML, Zhang DM, Li YH, Wang YF, Tao JY, Zhang SL,

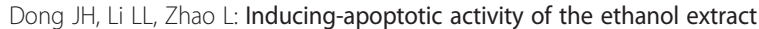
of Duchesnea indica Focke on treatment of herpes simplex encephalitis. Int J Immunopathol Pharmacol 2011, 24:631-638.

28. Zhao L, Tao JY, Zhang SL, Pang R, Jin F, Dong JH, Guo YJ: Inner antiinflammatory mechanisms of petroleum ether extract from Melilotus suaveolens Ledeb. Inflammation 2007, 30:213-223.

29. Zhao L, Tao JY, Zhang SL, Jin F, Pang R, Dong JH: N-butanol extract from melilotus suaveolens ledeb affects Pro- and anti-inflammatory cytokines and mediators. Evid Based Complement Alternat Med 2010, 7:97-106.

30. Rodríguez-Garay EA: Cholestasis: human disease and experimental animal models. Ann Hepatol 2003, 2:150-158

31. Golbar HM, Izawa T, Yano R, Ichikawa C, Sawamoto O, Kuwamura M, Lamarre J, Yamate J: Immunohistochemical characterization of macrophages and myofibroblasts in a-Naphthylisothiocyanate (ANIT)-induced bile duct injury and subsequent fibrogenesis in rats. Toxicol Pathol 2011, 39:795-808.

32. Roth RA, Dahm LJ: Neutrophil- and glutathione-mediated hepatotoxicity of alpha-naphthylisothiocyanate. Drug Metab Rev 1997, 29:153-165.

33. Mehendale HM, Roth RA, Gandolfi AJ, Klaunig JE, Lemasters JJ, Curtis LR: Novel mechanisms in chemically induced hepatotoxicity. FASEB J 1994, 8:1285-1295.

34. Zhao L, Zhang SL, Tao JY, Jin F, Pang R, Guo YJ, Ye P, Dong JH, Zheng GH: Anti-inflammatory mechanism of a folk herbal medicine, Duchesnea indica (Andr) Focke at RAW264.7 cell line. Immunol Invest 2008, 37:339-357.

35. Zhao L, Tao JY, Zhang SL, Jin F, Pang R, Dong JH, Guo YJ, Ye P: Anti-inflammatory mechanism of Rungia pectinata (Linn.) Nees. Immunopharmacol Immunotoxicol 2008, 30:135-151.

36. Aiken CT, Kaake RM, Wang X, Huang L: Oxidative stress-mediated regulation of proteasome complexes. Mol Cell Proteomics 2011, 10:R110.006924

37. van der Veen BS, de Winther MP, Heeringa P: Myeloperoxidase: molecular mechanisms of action and their relevance to human health and disease. Antioxid Redox Signal 2009, 11:2899-2937.
38. Hernández V, Miranda M, Pascual I, Sanchiz V, Almela P, Añón R, Cuadrado E, Sanz MI, Mínguez M, Mora F, Romero FJ, Benages A: Malondialdehyde in early phase of acute pancreatitis. Rev Esp Enferm Dig 2011, 103:563-569.

39. Buettner GR: Superoxide dismutase in redox biology: the roles of superoxide and hydrogen peroxide. Anticancer Agents Med Chem 2011, 11:341-346.

40. Assady M, Farahnak A, Golestani A, Esharghian M: Superoxide dismutase (SOD) enzyme activity assay in fasciola spp. Parasites and liver tissue extract. Iran J Parasitol 2011, 6:17-22

41. Phillips L, Toledo AH, Lopez-Neblina F, Anaya-Prado R, Toledo-Pereyra LH: Nitric oxide mechanism of protection in ischemia and reperfusion injury. $J$ Invest Surg 2009, 22:46-55.

42. Abe $Y$, Hines I, Zibari G, Grisham MB: Hepatocellular protection by nitric oxide or nitrite in ischemia and reperfusion injury. Arch Biochem Biophys 2009, 484:232-237.

43. González R, Cruz A, Ferrín G, López-Cillero P, Fernández-Rodríguez R, Briceño J, Gómez MA, Rufián S, Mata Mde L, Martínez-Ruiz A, Marin Jر, Muntané J: Nitric oxide mimics transcriptional and post-translational regulation during a-tocopherol cytoprotection against glycochenodeoxycholate-induced cell death in hepatocytes. 1 Hepatol 2011, 55:133-144.

44. Colombo R, de L Batista AN, Teles HL, Silva GH, Bomfim GC, Burgos RC, Cavalheiro AJ, da Silva Bolzani V, Silva DH, Pelícia CR, Guimarães FM, Heimberg MC: Validated HPLC method for the standardization of Phyllanthus niruri (herb and commercial extracts) using Corilagin as a phytochemical marker. Biomed Chromatogr 2009, 23:573-580.

45. Jikai L, Yue H, Henkel T, Weber K: One step purification of Corilagin and ellagic acid from Phyllanthus urinaria using high-speed countercurrent chromatography. Phytochem Anal 2002, 13:1-3.

doi:10.1186/1471-230X-13-79

Cite this article as: Jin et al:: Anti-inflammatory and anti-oxidative effects of corilagin in a rat model of acute cholestasis. BMC Gastroenterology 2013 13:79

\section{Submit your next manuscript to BioMed Central and take full advantage of:}

- Convenient online submission

- Thorough peer review

- No space constraints or color figure charges

- Immediate publication on acceptance

- Inclusion in PubMed, CAS, Scopus and Google Scholar

- Research which is freely available for redistribution 